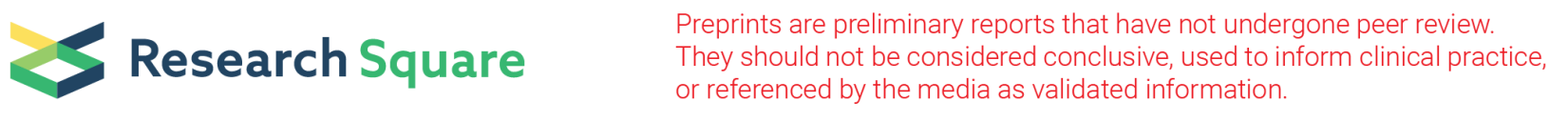

\title{
The Effect of Different Levels of Prostate-Specific Antigen and Prostate Imaging Report and Data System Scores on Logistic Regression Model to Predict Clinically Significant Prostate Cancer-A Single Center Retrospective Study
}

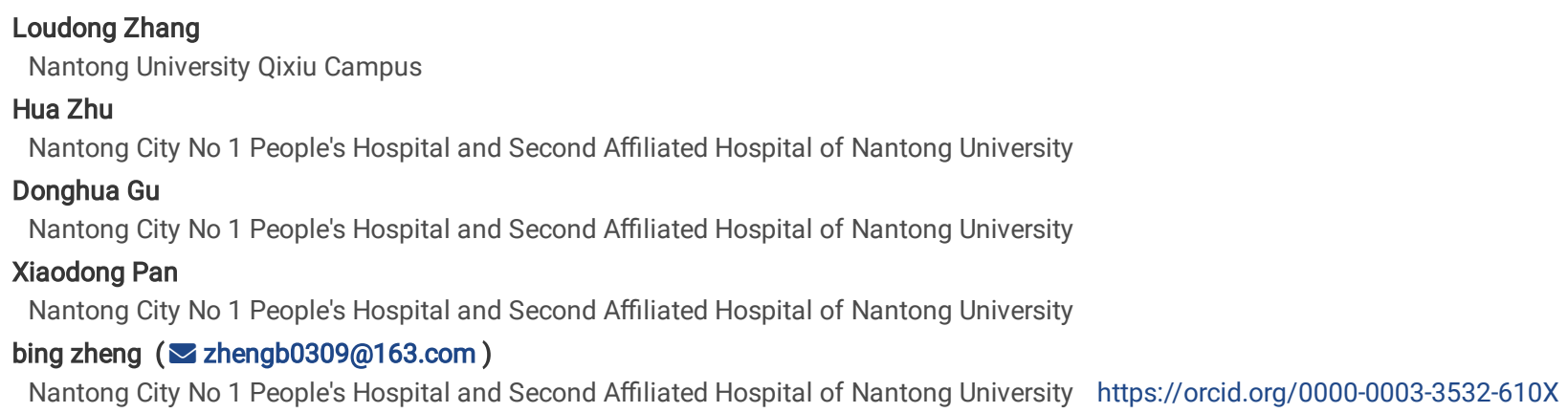




\section{Abstract}

Background: At present, there are various clinical regression models for predicting prostate cancer. But what about the diagnostic effectiveness of these models in different parameter ranges, and are the models applicable to everyone? This study aimed to study the influence of different levels of prostatespecific antigen (PSA) and Prostate Imaging Report and Data System version 2 (PI-RADS v2) scores on the regression model to predict clinically significant prostate cancer (csPCa).

Methods: This retrospective study screened 251 patients from our hospital, who were divided into different groups. The regression model was established for each group to predict csPCa, and the effects of PSA and PI-RADS scores on each model were analyzed through the diagnostic effects of the model.

Results: In patients with lower PSA scores, although the model was less sensitive than PSA, the AUC of the model was much greater. With the rise of PSA, the sensitivity of the model surpassed that of PSA, while the specificity became the opposite, and the AUC gap also gradually decreased. In the group with low PIRADS score, the sensitivity and specificity of PI-RADS were lower than the model, and the gap was larger. Although the gap between the two gradually decreased with the increase of PI-RADS, the diagnostic efficiency of the model was still slightly larger than that of pure PI-RADS.

Conclusion: As the PSA and PI-RADS v2 scores increase, the diagnostic advantages of the regression model will gradually decrease. However, for patients with Iow levels of PSA and PI-RADS scores,the regression model is less affected by PSA and PI-RADS, and can better utilize its clinical diagnostic advantages.

\section{Background}

Prostate cancer (PCa) is one of the most common cancers around the world and ranks among the top five in terms of morbidity and mortality (1). In clinical practice, however, most PCa require no more than regular follow-up and are unlikely to cause serious consequences even if left untreated. Prostate-specific antigen (PSA) is commonly applied in clinical practice to assist with the detection and screening of PCa. It is easily obtainable and simple to operate. However, it shows some disadvantages as well, for example, the lesion is incapable to be located and it tends to be affected by other prostate diseases.

Though prostate biopsy is regarded as the most authoritative standard for the diagnosis of PCa, it exhibits such drawbacks as low cancer detection rate, overdiagnosis, unnecessary invasive treatment, and increase in complications (2). In the meantime, there remain various difficulties in identifying clinically significant prostate cancer (csPCa). Therefore, it is a pressing need to detect csPCa and reduce the probability of over-diagnosis for low-risk diseases.

At present, multiparameter magnetic resonance imaging (mp-MRI) has received recognition as the most effective approach to imaging detection for the diagnosis of PCa. It is not only applicable for PCa detection, localization, partial stage and risk stratification, but also able to providing guidance on biopsy and clinical treatment $(3,4)$. It has been demonstrated in existing research that mp-MRI is capable to detect and locate csPCa lesions in a precise way $(5,6)$. In spite of this, the absence of a universal system image reporting and data system makes it difficult to measure the accuracy of mp-MRI for the diagnosis of PCa. The Prostate Imaging Report and Data System Version 2 (PI-RADS v2) updated in 2015 can provide a semi-quantitative criteria for the diagnosis of PCa (7).

However, the overall five-point scale used in PI-RADS v2 is not intended for all types of cancer, as it is applicable solely to csPCa, which may require further examination or targeted biopsy. As for the lesions with PI-RADS v2 $\geq 4$, the area under the curve (AUC) for the detection of clinically significant PCa is 0.79 (8). Meanwhile, it is difficult for the scoring system to determine the nature of lesions with a score of 3 . According to estimate, the negative predictive value of magnetic resonance imaging (MRI) excluding clinically significant cancers is no greater than $90 \%$, suggesting that MRI may be suitable as a tool to assist with screening. Nevertheless, MRI alone is not reliable when it is necessary to rule out major diseases (9).

Therefore, many studies have combined traditional risk factors with PI-RADS to predict csPCa through regression models, and have achieved certain results. But are these models suitable for all patients? How does each factor affect the model? This study aims to study the effect of different levels of PSA and PIRADS scores on the model, and compare the diagnostic capabilities of the models in different groups to find out the scope of the model.

\section{Materials And Methods}

Screening was conducted for the patients who were subject to MRI and prostate biopsy at our hospital between January 2017 and June 2019 . The inclusion criteria included increase in PSA levels, suspected MRI abnormalities, and digital rectal abnormalities. The exclusion criteria were as follows. The patients had a history of prostate biopsy, radical prostatectomy, and contraindications to TRUS-guided biopsy. There were 251 patients screened and divided into 5 different groups according to PSA or PI-RADS v2 scores. Group 1: $4 \mathrm{ng} / \mathrm{mL} \leq \mathrm{PSA}<10 \mathrm{ng} / \mathrm{mL}$; Group 2: $10 \mathrm{ng} / \mathrm{mL} \leq \mathrm{PSA}<20 \mathrm{ng} / \mathrm{mL}$; $\mathrm{Group} \mathrm{3:} 20 \mathrm{ng} / \mathrm{mL} \leq$ PSA < $200 \mathrm{ng} / \mathrm{mL}$; Group 4: PI-RADS v2 scores of 2, 3; Group 5: PI-RADS v2 scores of 4, 5. The characteristic of each group was shown in the Table 1. For each group, with the patient's PSA and PI-RADS v2 score as parameters, a model was constructed to make prediction of csPCa, from model A to model E. 
Table 1

Characteristics of the study population.

\begin{tabular}{|c|c|c|c|c|c|}
\hline & $\begin{array}{l}4 \mathrm{ng} / \mathrm{mL} \leq \mathrm{PSA}< \\
10 \mathrm{ng} / \mathrm{mL}\end{array}$ & $\begin{array}{l}10 \mathrm{ng} / \mathrm{mL} \leq \mathrm{PSA}< \\
20 \mathrm{ng} / \mathrm{mL}\end{array}$ & $\begin{array}{l}20 \mathrm{ng} / \mathrm{mL} \leq \mathrm{PSA}< \\
200 \mathrm{ng} / \mathrm{mL}\end{array}$ & $\begin{array}{l}\text { PI-RADS v2: } 2 \text { and } 3 \\
\text { scores }\end{array}$ & $\begin{array}{l}\text { PI-RADS v2: } 4 \text { and } 5 \\
\text { scores }\end{array}$ \\
\hline Number of patients & 71 & 83 & 97 & 56 & 195 \\
\hline Age (mean \pm SD) & $69.14 \pm 9.20$ & $72.79 \pm 8.18$ & $72.84 \pm 8.98$ & $64.95 \pm 8.07$ & $72.37 \pm 9.03$ \\
\hline $\begin{array}{l}\text { PSA levels (mean } \\
\pm \text { SD) }\end{array}$ & $7.02 \pm 1.98$ & $14.27 \pm 2.85$ & $52.35 \pm 10.31$ & $14.11 \pm 8.29$ & $30.81 \pm 16.90$ \\
\hline \multicolumn{6}{|l|}{ PI-RADS v2 } \\
\hline 2 & 3 & 2 & 1 & 6 & - \\
\hline 3 & 23 & 19 & 8 & 50 & - \\
\hline 4 & 38 & 41 & 43 & - & 122 \\
\hline 5 & 7 & 21 & 45 & - & 73 \\
\hline Insignificant PCa & 11 & 15 & 7 & 8 & 25 \\
\hline Significant PCa & 20 & 30 & 65 & 7 & 108 \\
\hline
\end{tabular}

The study was approved by the institutional ethics committee of the Second Affiliated Hospital of Nantong University. Informed consents were obtained from participants in the study.

\section{Biopsy procedure}

When biopsy was conducted, a 12-needle systematic biopsy was first performed through the rectum, prior to a $3 \pm 5$ needle targeted biopsy carried out by the same operator. All biopsy tissues were immediately fixed in formalin for the subsequent routine histopathological evaluation.

\section{Statistical analysis}

SPSS 20.0 and Medical statistical software were applied to process the data, while PI-RADS v2 and PSA were fitted by conducting Logistic regression analysis to establish the corresponding model. The receiver operating characteristic curve was used to conduct an evaluation of the model for its diagnostic efficacy of predicting csPCa. The closer the AUC was to 1, the higher the diagnostic accuracy was. Quantitative variables like PSA and PI-RADS v2 scores were expressed as means and standard deviations. Furthermore, $Z$ test was conducted to compare the area under different curves. According to the test level of 0.05 , $p<0.05$ was treated as statistically significant.

\section{Results}

In this study, a total of 251 patients underwent cognitive fusion biopsy, with an average age of $71.71 \pm 8.92$ and a mean PSA of $27.11 \pm 20.57$. Among them, 103 patients were non-PCa, and 115 patients were diagnosed with csPCa. The overall positive rate was $58.96 \%$, and the detection rate was $45.81 \%$ for csPCa. The comparison of diagnostic efficacy among PSA, PI-RADS and models was shown in the Table 2.

Table 2

Comparison of diagnostic efficacy among PSA, PI-RADS and models.

\begin{tabular}{|c|c|c|c|c|c|c|c|c|c|c|}
\hline & \multicolumn{4}{|c|}{$4 \mathrm{ng} / \mathrm{mL} \leq \mathrm{PSA}<10 \mathrm{ng} / \mathrm{mL}$} & \multicolumn{4}{|c|}{$10 \mathrm{ng} / \mathrm{mL} \leq \mathrm{PSA}<20 \mathrm{ng} / \mathrm{mL}$} & \multicolumn{2}{|c|}{$20 \mathrm{ng} / \mathrm{mL} \leq \mathrm{PSA}<$} \\
\hline & PSA & PI-RADS v2 & Model A & $\begin{array}{l}\mathrm{p} \\
\text { value }\end{array}$ & PSA & PI-RADS v2 & Model B & $\begin{array}{l}\mathrm{p} \\
\text { value }\end{array}$ & PSA & $\mathrm{F}$ \\
\hline Sensitivity & 0.698 & 0.634 & 0.636 & & 0.419 & 0.484 & 0.677 & & 0.538 & 0 \\
\hline Specificity & 0.500 & 0.613 & 0.875 & & 0.867 & 0.853 & 0.800 & & 0.859 & 0 \\
\hline \multirow[t]{3}{*}{$\begin{array}{l}\text { AUG }(95 \% \\
\mathrm{Cl})\end{array}$} & $\begin{array}{l}0.528 \\
(0.243-0.814)\end{array}$ & $\begin{array}{l}0.785 \\
(0.611-0.979)\end{array}$ & $\begin{array}{l}0.747 \\
(0.607-0.986)\end{array}$ & 0.040 & $\begin{array}{l}0.533 \\
(0.362-0.704)\end{array}$ & $\begin{array}{l}0.714 \\
(0.557-0.871)\end{array}$ & $\begin{array}{l}0.744 \\
(0.592-0.896)\end{array}$ & 0.037 & $\begin{array}{l}0.732 \\
(0.591-0.873)\end{array}$ & $\begin{array}{l}0 \\
(1\end{array}$ \\
\hline & \multicolumn{4}{|c|}{ PI-RADS v2: 2 and 3 scores } & \multicolumn{4}{|c|}{ PI-RADS v2: 4 and 5 scores } & & \\
\hline & PSA & PI-RADS v2 & Model D & $\begin{array}{l}\mathrm{p} \\
\text { value }\end{array}$ & PSA & PI-RADS v2 & Model E & $\mathrm{p}$ value & & \\
\hline Sensitivity & 0.552 & 0.421 & 0.556 & & 0.537 & 0.554 & 0.669 & & & \\
\hline Specificity & 0.880 & 0.730 & 0.892 & & 0.812 & 0.826 & 0.818 & & & \\
\hline $\begin{array}{l}\text { AUG }(95 \% \\
\mathrm{Cl})\end{array}$ & $\begin{array}{l}0.750 \\
(0.509-0.991)\end{array}$ & $\begin{array}{l}0.625 \\
(0.348-0.902)\end{array}$ & $\begin{array}{l}0.833 \\
(0.640-1.000)\end{array}$ & 0.045 & $\begin{array}{l}0.650 \\
(0.557-0.744)\end{array}$ & $\begin{array}{l}0.686 \\
(0.574-0.798)\end{array}$ & $\begin{array}{l}0.731 \\
(0.638-0.824)\end{array}$ & 0.132 & & \\
\hline
\end{tabular}

The patients were split into 3 different groups according to PSA: $4 \mathrm{ng} / \mathrm{mL} \leq \mathrm{PSA}<10 \mathrm{ng} / \mathrm{mL}, 10 \mathrm{ng} / \mathrm{mL} \leq \mathrm{PSA}<20 \mathrm{ng} / \mathrm{mL}, 20 \mathrm{ng} / \mathrm{mL} \leq \mathrm{PSA}<200 \mathrm{ng} / \mathrm{mL}$ 
$4 \mathrm{ng} / \mathrm{mL} \leq P S A<10 \mathrm{ng} / \mathrm{mL}, Y=\operatorname{Lgit}(P)=-7.389+2.688 \cdot P I-R A D S-0.227 \cdot P S A$

There were 71 patients in the group 1, 20 patients were diagnosed with csPCa. In this group of patients, the threshold for model A was 0.815 . When csPCa was predicted, the specificity (0.875) of model A was superior to PI-RADS v2 (0.613) and PSA (0.500), despite the sensitivity (0.636) being slightly lower than PSA (0.698). Among them, the highest AUC was PI-RADS v2 (0.785). The AUC of PSA and model A were 0.528 and 0.747 , respectively. The difference was of statistical significance.

$10 \mathrm{ng} / \mathrm{mL} \leq P S A<20 \mathrm{ng} / \mathrm{mL}, Y=\operatorname{Lgit}(P)=-4.574+1.616 \cdot P I-R A D S-0.099 \cdot P S A$

In the group 2, 30 of 83 people were diagnosed with csPCa. According to the prediction of csPCa, The sensitivity of model B was (0.677), which was significantly better than PSA and PI-RADS v2. and the threshold was 0.647 , while the level of specificity was 0.800 . The AUC of the model B was 0.744 , which suggested a decent level of accuracy. And the AUC of clinically significant PCa was 0.714 and 0.533 respectively as predicted by PI-RADS v2 and PSA, whose diagnostic efficacy was inferior to the model B. The $p$ value was 0.037 .

$20 \mathrm{ng} / \mathrm{mL} \leq P S A<200 \mathrm{ng} / \mathrm{mL}, Y=L g i t(P)=-2.761+0.6955 \cdot P I-R A D S+0.045 \cdot P S A$

The group 3 contained 97 patients, 65 of whom were diagnosed with csPCa. With the increase of PSA, the specificity and threshold of model $C$ were increased to 0.857 and 0.901 , while the sensitivity was merely 0.615 , which was slightly higher compared to PI-RADS v2 (0.600). The AUC of the model C was 0.769 , which was higher than PI-RADS v2 and PSA, despite no significant difference among them.

According to PI-RADS v2, patients with 2 or 3 scores were assigned to one group, while those with 4 or 5 scores were assigned into the other group

PI-RADS: 2 and 3 scores, $Y=L$ git $(P)=-64.286+21.141 \cdot P I-R A D S+0.081 \cdot P S A$

In this group, only 7 out of 56 people were found csPCa. Compared with pure PI-RADS, Model D had obvious advantages in predicting csPCa. Its threshold was 0.658 , and its sensitivity and specificity were 0.556 and 0.892 respectively, which were greater than PI-RADS $(0.412,0.73)$. The AUC of the model was 0.833 , which might indicate that the model was less affected by PI-RADS and had a better predictive effect on csPCa.

PI-RADS: 4 and 5 scores, $Y=\operatorname{Lgit}(P)=-5.112+1.405 \cdot P I-R A D S+0.023 \cdot P S A$

In the group 5, 108 of the 195 patients had csPCa detected. The threshold of model E was 0.838 , and the sensitivity was 0.669 . As the PI-RADS score increased, the model was gradually affected by PI-RADS, and the gap in diagnostic performance was gradually narrowed. In addition, the comparison of AUC was 0.731 (model E) vs 0.686 (PI-RADS v2) vs 0.650 (PSA). The difference was not statistically significant.

\section{Discussion}

At present, the imaging evaluation of the prostate using mp-MRI of the PI-RADS v2 classification standard has played a crucial role in the screening and evaluation of the patients suspected of having PCa. It has been proven to be capable of accurate detection of PCa and is closely associated with confirmatory pathological analysis based on the Gleason classification system (10-12). In a meta-analysis of PI-RADS v2 diagnostic performance studies, Woo et al. reported the sensitivity and specificity of 0.89 and 0.73 , respectively (13). In another meta-analysis, the sensitivity and specificity of PI-RADS v2 reached 0.85 and 0.71 , respectively (14). Both meta-analyses demonstrated a high level of sensitivity and a moderate level of specificity.

As revealed by most studies evaluating the cancer detection rate of PI-RADS v2, the higher the level of lesions detected by PI-RADS v2, the higher the cancer detection rate $(7,8,15,16)$. Nevertheless, some studies have also concluded that the correlation is relatively insignificant. According to the findings of Mehralivand, among the lesions with a PI-RADS v2 score of 5, the detection rate of cancer was the highest (86.9\%), but the lesions with a score of 4 were merely $39.1 \%$, which contradicted the definition of "possible clinical cancer" in a 4-score lesion (17).

In this study, imaging evaluation was conducted again on the lesions with negative puncture in some PI-RADS v2 score 4 cases. Without knowing it, 5 radiologists were asked to reassess and discuss 38 cases with a score of 4 , and some of which were re-rated to 3 scores. Since the lesion grade was evaluated by the radiologists, the results might be subject to the impact of cognitive bias, and there might be underestimation or overestimation of the lesion grade for other lesions, which made it difficult to identify the patients who could avoid or receive puncture by PI-RADS v2 score alone. As for this part of the re-evaluated patients, no second puncture was performed, as a result of which it was impossible to judge whether there was any penetration of suspected lesions missed during the puncture process and the accuracy of re-assessment.

The combination of available molecules and traditional risk factors in the Logistic regression model was conducted to determine their relative contributions and significance to predicting the clinical feasibility of csPCa. In order to improve the detection rate of cancer, Dikaios et al. proposed the application of mpMRI to construct Logistic model for the classification of csPCa (18). Niu et al. believed that the model was effective in reducing the damage caused by unnecessary prostate biopsy while predicting high-risk PCa (19). By incorporating age and PI-RADS v2 score, they adjusted PSAD into the model, based on which a better AUC of 0.86 was achieved. The sensitivity and specificity of the model reached $83.3 \%$ and $87.4 \%$, respectively.

In our research, the PSA and PI-RADS v2 were applied to predict csPCa by Logistic model fitting, and an evaluation was conducted of the model for its diagnostic efficacy in each group. Although the AUC of almost all models was greater than PSA and PI-RADS v2, the diagnostic efficiency of the models was not necessarily better than PSA and PI-RADS. 
In the PSA group, when PSA was low, the specificity and AUC of model A were significantly better than PSA. However, with the increase of PSA, the diagnostic efficiency of the two was gradually approaching, which meant that the increase of PSA leaded to a greater impact on the model. This showed that the value of using the model in patients with high PSA levels was not high, and for patients with low PSA, using this model could effectively reduce the impact of other prostate diseases and improve the detection of csPCa. Figure 1 compared the AUC of the PSA group.

In the PI-RADS group, the diagnostic advantages of models D and E were different in each group, and changed with the increase of PI-RADS. However, because of the small number of patients with PI-RADS 2 scores when screening patients, the diagnostic efficacy of Model D might exceed the actual value. Figure 2 showed the comparison of the AUC of the PI-RADS group.

Despite plenty of studies where new promising PCa-specific biomarkers have been discovered, there are only a few biomarkers which have reached the stage of clinical application so far. Leander Van added HOXC6 and DLX1 mRNA levels to the clinical risk factor model, but failed to make significant improvement to AUC (20). In the view of Cantiello, both prostate health index (PHI) and prostate cancer gene 3 (PCA3) could improve the prediction of PCa, and PHI significantly improved the accuracy of 7.9\% (21). However, there were some studies indicating that PCA3 had only limited predictive power (22-24). In addition, in multiple Logistic regression analyses, PCA3 was not an independent risk factor for the more aggressive PCa.

According to the results of this paper, it is of significance to combine useful predictors to construct a model for improving the diagnostic ability and facilitating PCa classification. However, the efficacy of the model in each group varies, which makes it necessary to determine the applicable scope of the model and to identify the appropriate indicators for guiding prostate biopsy.

The following limitation is found out in this study. Due to the small sample size, our results may differ from other researches to some extent. In addition, because of the lack of data, the new model ignores certain parameters, for example, body mass index and other biochemical indexes. Finally, the current model has not yet been validated on an external data set, as a result of which further testing and validation are required in more centers with larger samples.

\section{Conclusion}

With PSA and PI-RADS v2 used as component parameters, the regression model can ensure the accurate detection of csPCa, which can assist with clinical decision-making while improving the outcome of diagnosis and management for PCa patients. Nevertheless, the application of the model tends to be affected by PSA and PI-RADS v2 scores, especially when the two are at a higher level. Although the diagnostic efficiency of the model has improved, its advantages are gradually declining.

\section{Abbreviations}

PSA

prostate-specific antigen; PCa:Prostate cancer; csPCa:clinically significant prostate cancer; mp-MRI:multiparameter magnetic resonance imaging; PI-RADS v2:Prostate Imaging Report and Data System Version 2; AUC:area under the curve.

\section{Declarations}

Acknowledgements Not applicable

Authors' contributions LDZ was involved in literature search as well as data acquisition and analysis. HZ, DHG and XDP were the attending physicians responsible for carrying out the bioptic procedures during the surgery. All authors contributed to result interpretation and manuscript drafting.

Funding This research did not receive any specific grants from funding agencies in the public, commercial, or not-for-profit sectors.

Availability of data and material All data are available from the corresponding author on reasonable request.

Ethics approval The study was approved by the Ethics Committee of The Second Affiliated Hospital of Nantong University.

Consent for publication Not obtained, due to the retrospective character of the study.

Conflict of interest The authors declare that they have no conflict of interest.

\section{References}

1. Ferlay J, Soerjomataram I, Dikshit R, et al. Cancer incidence and mortality worldwide: sources, methods and major patterns in GLOBOCAN 2012. Int J Cancer. 2015;136(5):E359-86.

2. Donovan JL, Hamdy FC, Lane JA, et al. Patient-reported outcomes after monitoring, surgery, or radiotherapy for prostate cancer. N Engl J Med. 2016;375(15):1425-37.

3. Ho R, Siddiqui MM, George AK, et al. Preoperative multiparametric magnetic resonance imaging predicts biochemical recurrence in prostate cancer after radical prostatectomy. PLoS One. 2016;11(6):e0157313.

4. Updated Prostate Imaging Reporting and Data System (PIRADS

Vargas HA, Hotker AM, Goldman DA, et al. Updated Prostate Imaging Reporting and Data System (PIRADS v2) recommendations for the detection of clinically significant prostate cancer using multiparametric MRI: critical evaluation using whole-mount pathology as standard of reference. Eur Radiol 
2016; 26 (6): 1606-12.

5. Radtke JP, Schwab C, Wolf MB, et al. Multiparametric magnetic resonance imaging (MRI) and MRI-transrectal ultrasound fusion biopsy for index tumor detection: correlation with radical prostatectomy specimen. Eur Urol. 2016;70(5):846-53.

6. Ahmed HU, El-Shater Bosaily A, Brown LC, et al. Diagnostic accuracy of multi-parametric MRI and TRUS biopsy in prostate cancer (PROMIS): a paired validating confirmatory study. Lancet. 2017;389(10071):815-22.

7. Rosenkrantz AB, Babb JS, Taneja SS, Ream JM. Proposed adjustments to PI-RADS version 2 decision rules: Impact on prostate cancer detection. Radiology. 2017;283(1):119-29.

8. Park SY, Jung DC, Oh YT, et al. Prostate cancer: PI-RADS version 2 helps preoperatively predict clinically significant cancers. Radiology. 2016;280(1):10816.

9. Itatani R, Namimoto T, Atsuji S, et al. Negative predictive value of multiparametric MRI for prostate cancer detection: outcome of 5-year follow-up in men with negative findings on initial MRI studies. Eur J Radiol. 2014;83(10):1740-5.

10. Cash H, Maxeiner A, Stephan C, et al. The detection of significant prostate cancer is correlated with the prostate imaging reporting and data system (PIRADS) in MRI/transrectal ultrasound fusion biopsy. World J Urol. 2016;34(4):525-32.

11. Baldisserotto M, Neto EJ, Carvalhal G, et al. Validation of PI-RADS v.2 for prostate cancer diagnosis with MRI at 3T using an external phased-array coil. J Magn Reson Imaging. 2016;44(5):1354-9.

12. Kasel-Seibert M, Lehmann T, Aschenbach R, et al. Assessment of PI-RADS v2 for the detection of prostate cancer. Eur J Radiol. 2016;85(4):726-31.

13. Woo S, Suh CH, Kim SY, Cho JY, Kim SH. Diagnostic performance of Prostate Imaging Reporting and Data System Version 2 for detection of prostate cancer: a systematic review and diagnostic meta-analysis. Eur Urol. 2017;72(2):177-88.

14. A meta-analysis of use of Prostate Imaging Reporting and Data System Zhang L, Tang M, Chen S, Lei X, Zhang X, Huan Y. A meta-analysis of use of Prostate Imaging Reporting and Data System Version 2 (PI-RADS V2) with multiparametric MR imaging for the detection of prostate cancer. Eur Radiol 2017; 27 (12): 5204-14.

15. Mehralivand S, Bednarova S, Shih JH, et al. Prospective evaluation of PI-RADS Version 2 using the International Society of Urological Pathology Prostate Cancer Grade Group System. J Urol. 2017;198(3):583-90.

16. Muller BG, Shih JH, Sankineni S, et al. Prostate cancer: interobserver agreement and accuracy with the revised Prostate Imaging Reporting and Data System at multiparametric MR imaging. Radiology. 2015;277(3):741-50.

17. PI-RADS Prostate Imaging - Reporting and Data System: 2015, Version 2

Weinreb JC, Barentsz JO, Choyke PL, et al. PI-RADS Prostate Imaging - Reporting and Data System: 2015, Version 2. Eur Urol 2016 ; 69 (1): $16-40$.

18. Dikaios N, Alkalbani J, Abd-Alazeez M, et al. Zone-specific logistic regression models improve classification of prostate cancer on multi-parametric MRI. Eur Radiol. 2015;25(9):2727-37.

19. Niu XK, He WF, Zhang Y, et al. Developing a new PI-RADS v2-based nomogram for forecasting high-grade prostate cancer. Clin Radiol. 2017;72(6):458-64.

20. Van Neste L, Hendriks RJ, Dijkstra S, et al. Detection of high-grade prostate cancer using a urinary molecular biomarker-based risk score. Eur Urol. 2016;70(5):740-8.

21. Cantiello F, Russo GI, Ferro M, et al. Prognostic accuracy of Prostate Health Index and urinary Prostate Cancer Antigen 3 in predicting pathologic features after radical prostatectomy. Urol Oncol. 2015;33(4):163 e115-23.

22. Auprich M, Chun FK, Ward JF, et al. Critical assessment of preoperative urinary prostate cancer antigen 3 on the accuracy of prostate cancer staging. Eur Urol. 2011;59(1):96-105.

23. Durand X, Xylinas E, Radulescu C, et al. The value of urinary prostate cancer gene 3 (PCA3) scores in predicting pathological features at radical prostatectomy. BJU Int. 2012;110(1):43-9.

24. Ploussard G, Durand X, Xylinas E, et al. Prostate cancer antigen 3 score accurately predicts tumour volume and might help in selecting prostate cancer patients for active surveillance. Eur Urol. 2011;59(3):422-9.

\section{Figures}
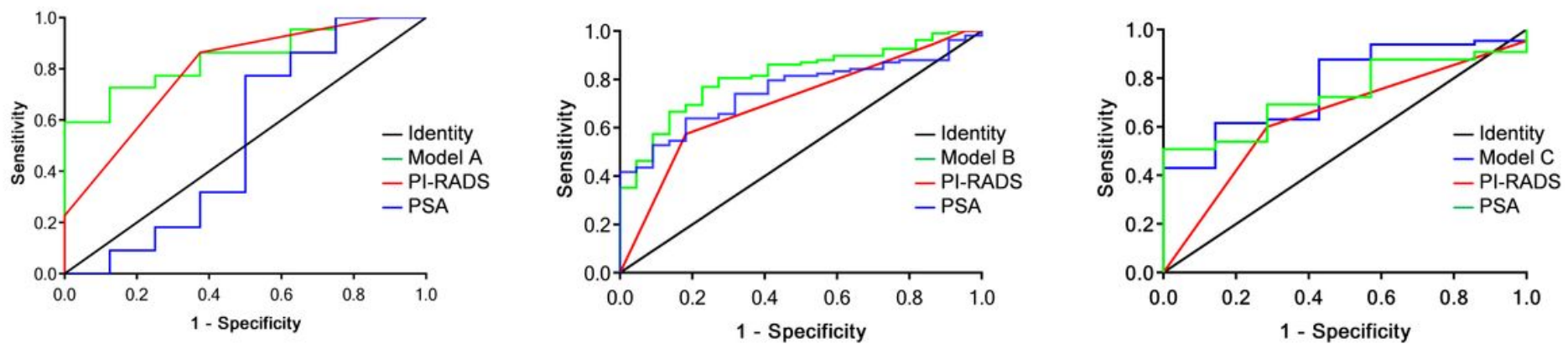

Figure 1

Page 6/7 
In the PSA group, the AUC of models A, B, and C was 0.747 vs 0.744 vs 0.769 . When the changes in PI-RADS v2 were not considered, as the level of PSA rose, the advantages of using the model gradually declined, although the diagnostic efficacy of the models had not changed.
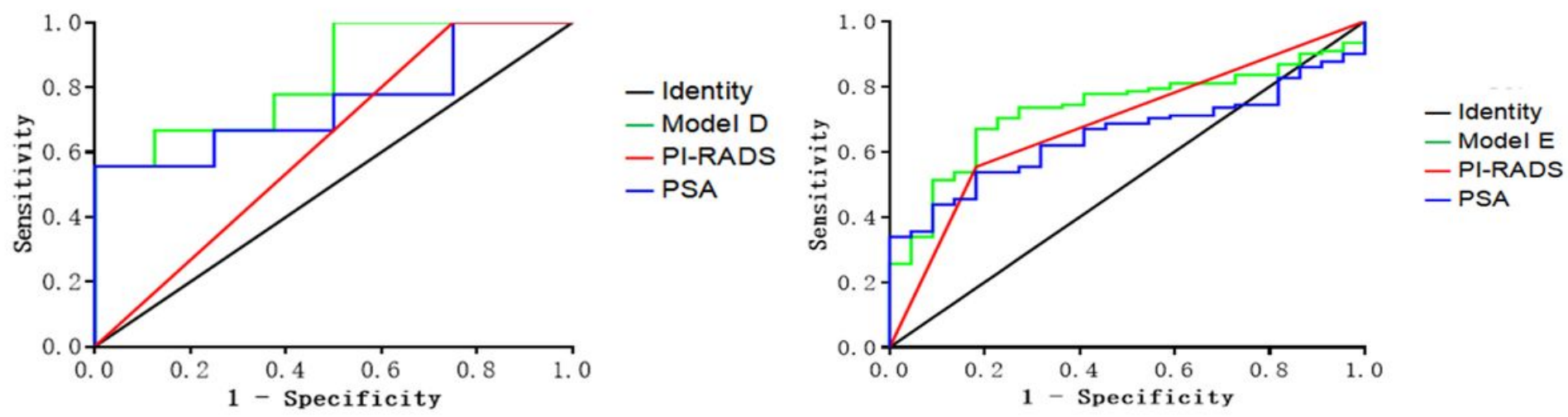

\section{Figure 2}

When grouped by PI-RADS v2, the AUC of models D and E was 0.833 vs 0.731 . Due to the definition of PI-RADS v2, the probability of detecting csPCa in group of 2 and 3 scores was lower than group of 4 and 5 scores. Therefore, the use of models in patients with low scores was more clinically meaningful. 